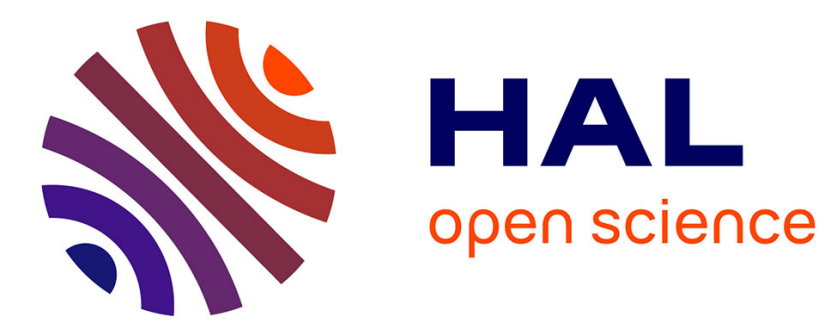

\title{
Hybrid Turbo Equalization for Faster-than-Nyquist Underwater Communication Systems
}

\author{
Tarak Arbi, Benoit Geller
}

\section{To cite this version:}

Tarak Arbi, Benoit Geller. Hybrid Turbo Equalization for Faster-than-Nyquist Underwater Communication Systems. IEEE Oceans 21, Sep 2021, San Diego, United States. hal-03426889

\section{HAL Id: hal-03426889 \\ https://hal.science/hal-03426889}

Submitted on 12 Nov 2021

HAL is a multi-disciplinary open access archive for the deposit and dissemination of scientific research documents, whether they are published or not. The documents may come from teaching and research institutions in France or abroad, or from public or private research centers.
L'archive ouverte pluridisciplinaire HAL, est destinée au dépôt et à la diffusion de documents scientifiques de niveau recherche, publiés ou non, émanant des établissements d'enseignement et de recherche français ou étrangers, des laboratoires publics ou privés. 


\section{Hybrid Turbo Equalization for Faster-than-Nyquist Underwater Communication Systems}

\author{
$1^{\text {st }}$ Tarak Arbi \\ Department U2IS, ENSTA Paris \\ Institut Polytechnique de Paris, France \\ tarak.arbi@ensta-paris.fr
}

\author{
$2^{\text {nd }}$ Benoit Geller \\ Department U2IS, ENSTA Paris \\ Institut Polytechnique de Paris, France \\ benoit.geller@ensta-paris.fr
}

\begin{abstract}
Faster-Than-Nyquist (FTN) signaling is a promising technique to enhance the UnderWater Acoustic (UWA) communications rate. However, the main issue of this technique is InterSymbol Interference (ISI) that needs to be tackled efficiently at the receiver side. To face this issue, we propose a new Hybrid Time-Frequency Domain Equalizer (HTDFE) for FTN UWA communication systems. In contrast to the conventional HTDFE, each Frequency Domain Equalizer (FDE) at the multiple output side is followed by a joint phase estimator and feedforwardfeedback Time Domain Equalizer (TDE). Simulations over a real underwater channel show that the proposed algorithm allows a gain in performance compared to the conventional solutions.

Index Terms-Faster-than-Nyquist (FTN), Inter-Symbol Interference (ISI), Time-Frequency Domain Equalizer (HTDFE), Frequency Domain Equalizer (FDE), Time Domain Equalizer (TDE), Turbo equalization, phase estimation.
\end{abstract}

\section{INTRODUCTION}

The UnderWater Acoustic (UWA) communication rate is severely limited due to the available bandwidth of the UWA channel [1]. Therefore, high spectral efficiency communications techniques, such as high-order modulation and Signal Space Diversity (SSD) [2]-[7], are very attractive for UWA communication systems; in particular, Fasterthan-Nyquist (FTN) signaling is an efficient technique for enhancing the transmission rate beyond that defined by the Nyquist criterion [8], [9]. Indeed, FTN signaling sends more symbols than the classical Nyquist signaling in a same time period without imposing any bandwidth expansion.

This leads inevitably to Inter-Symbol Interference (ISI) which has to be addressed effectively so as to obtain a good system performance. In particular, Time Domain Equalization (TDE) can considerably enhance the system performance [11]-[18]; however, for the UWA channel, the channel delay spread can be large, leading to a high computational complexity at the receiver side. To face this issue, Frequency Domain Equalization (FDE) has been proposed [18]-[20], as it allows rather good system performance with lower computational complexity. To further improve the system performance over long channel delay spreads such as encountered in UWA communications, Hybrid Time-Frequency Domain Equalizers (HTFDE) have been considered recently [21]-[24], where the complexity of the time-frequency equalizer is considerably lower than that of the time only equalizer while both techniques have similar system performance.

In this paper, a new multi-channel HTFDM equalizer for Faster-than-Nyquist UWA communications systems is proposed. It is based on several Minimum Mean Square Error (MMSE) FDE equalizers operating in parallel; each Frequency Domain Equalizer at the multiple output side is followed by a joint phase synchronizer and Feedforward-Feedback time domain equalizer. Simulations over different real underwater channels show that the proposed algorithm allows a gain in performance compared to the conventional solutions.

The reminder of this paper is organised as follows. Section II introduces the system model. In Section III, we detail the proposed HTFDM equalizer. Some numerical results are presented in Section V, and finally Section VI concludes the paper.

\section{SYSTEM MODEL}

Information bits $b$ are first channel encoded [25], [26], then interleaved, and finally mapped into M-QAM complex symbols. Thereafter, the symbols are processed in blocks of $\mathrm{N}$ symbols. A Pseudo Noise (PN) sequence header of length $P$, is then added at the beginning of each block (see Fig.1). The $l$-th block is presented by:

$$
s^{l}=\left[s^{l}(0), s^{l}(1), \cdots, s^{l}(N-1)\right]^{T},
$$

where $s^{l}(j)$ is the j-th QAM symbol of the block of index $l$. For the sake of simplicity and without loss of generality, the block index $l$ is dropped in the sequel of this paper. The transmitted symbols can be written as:

$$
s(t)=\sum_{n=0}^{N-1} s_{n} g(t-n T),
$$

where $g(t)$ is a shaping filter and $T=\alpha T_{0}$ is the symbol duration, where $T_{0}$ is the classical Nyquist bound of an ISIfree symbol duration and $\alpha$ is the so-called acceleration factor $0<\alpha \leq 1$ [9].

In this paper, we consider a Single-Input Multiple-Output (SIMO) configuration with $K$ receive antennas. At the receiver 


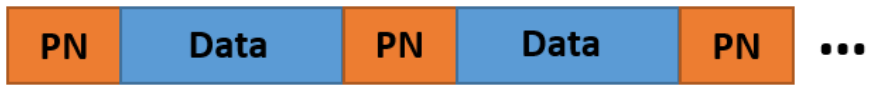

Fig. 1. Packet structure.

side, the receiver starts by separating the received symbols into blocks of $N$ observations. Assuming perfect time synchronization [27]-[30] and that the channel is invariant over each block duration, the received observation on the $k$-th channel at time $n$ can be written as:

$$
r_{k}(n)=e^{j \phi_{k, n}} \sum_{l=0}^{L-1} \sum_{m=-v}^{v} s_{n} h_{l}^{k} g(n T-(l+m) T)+w(n T),
$$

where $w$ is the Additive White Gaussian Noise (AWGN) of variance $\sigma_{w}^{2}, h_{l}^{k}$ indicates the channel response, $L$ is the channel length and $2 v$ is the number of ISI taps introduced by the FTN signaling.

Furthermore, both the phase variation and the ISI caused by the channel need to be combated efficently at the receiver side so as to achieve good performance. The proposed hybrid algorithm for joint equalization and phase estimation is detailed in the next section. In Fig.2, we obtain after hybrid time-frequency domain equalization, the symbols $z_{k}$. They can be expressed as:

$$
z_{k}=\beta_{0} s_{k}+\nu_{k},
$$

where $\beta_{0}$ is a constant bias factor $\beta_{0}$ and $\nu_{k}$ is a Gaussian noise of variance $\sigma_{v}^{2}$. The obtained symbols $z_{k}$ are fed into the Symbol to Bit Converter (SBC) in order to compute the Log-Likelihood Ratio (LLR) for each mapped bit:

$$
L L R\left(l^{i}\left(z_{k}\right)\right)=\ln \frac{\sum_{s \in \boldsymbol{S}_{i}^{1}} \exp \left(-\frac{\left|z_{k}-\beta_{0} s\right|^{2}}{\sigma_{v}^{2}}\right)}{\sum_{s \in \boldsymbol{S}_{i}^{0}} \exp \left(-\frac{\left|z_{k}-\beta_{0} s\right|^{2}}{\sigma_{v}^{2}}\right)},
$$

where $l^{i}\left(z_{k}\right)$ designates the $i$-th bit of symbol $z_{k}$ and $\boldsymbol{S}_{i}^{b}$ denotes the set of the constellation points whose $i$-th bit is $b=1$ or 0 .

Then, the channel decoder is applied; its extrinsic soft information output is used by the Bit to Symbol Converter (BSC) to obtain the soft value $\bar{s}_{k}^{(p-1)}$, for $p>1$. Finally, the symbols $\bar{s}_{k}^{(p-1)}$ are used in the next iteration $p$ of our proposed algorithm so as to enhance the equalization performance. Finally, after $p_{\max }$ iterations (i.e. equalization/channel decoder), a hard decision on the LLRs at the output of the decoder is made to estimate the user information bits.

\section{THE PROPOSED HYBRID TURBO EQUALIZATION TECHNIQUE}

We now describe the proposed joint equalization and phase error estimation algorithm depicted in Fig. 2. For each received antenna, the receiver performs equalization in the frequency domain followed by equalization and phase synchronisation

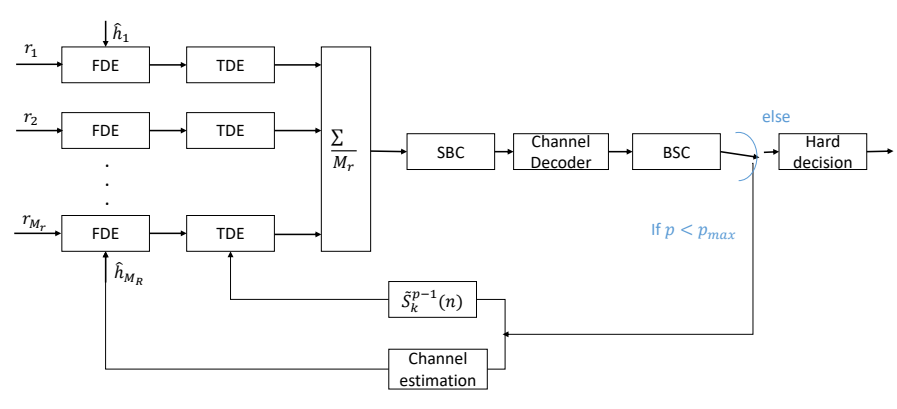

Fig. 2. Proposed architecture.

in the time domain. The following subsections present the components of the proposed hybrid equalizer.

\section{A. Frequency Domain Equalizer (FDE)}

Eq. 3 represents a circular-matrix based structure, so that the $k$-th channel received symbol block of length $N+P$ can be rewritten as:

$$
\boldsymbol{r}_{k}=\boldsymbol{H}_{\boldsymbol{k}} \boldsymbol{s}+\boldsymbol{w}_{\boldsymbol{k}},
$$

where $\boldsymbol{H}$ is a circulant matrix having an eigen-decomposition:

$$
\boldsymbol{H}_{\boldsymbol{k}}=\boldsymbol{Q}^{T} \boldsymbol{\Delta}_{\boldsymbol{k}} \boldsymbol{Q}^{*},
$$

where $Q$ is the Fourier transform matrix.

The received symbol in the frequency domain can be written as:

$$
\begin{aligned}
\boldsymbol{R}_{\boldsymbol{k}} & =\boldsymbol{Q}^{*} \boldsymbol{r}_{\boldsymbol{k}}, \\
& =\boldsymbol{\Delta}_{k} \boldsymbol{Q}^{*} \boldsymbol{s}+\boldsymbol{Q}^{*} \boldsymbol{w}_{\boldsymbol{k}}, \\
& =\boldsymbol{\Delta}_{\boldsymbol{k}} \boldsymbol{S}+\boldsymbol{W}_{\boldsymbol{k}},
\end{aligned}
$$

where $\boldsymbol{R}_{\boldsymbol{k}}$ (resp. $\boldsymbol{W}_{\boldsymbol{k}}$ ) designates the symbol block (resp. the Gaussian noise) in the frequency domain. Using the MMSE criterion [19], the receiver obtains the equalized symbol in the frequency domain:

$$
\tilde{z}_{k}=M_{k} \boldsymbol{R}_{k}
$$

where $M_{k}$ is a diagonal matrix whose i-th element is equal to:

$$
\boldsymbol{M}_{\boldsymbol{k}}(i)=\frac{\lambda_{k}^{*}(i)}{\left|\lambda_{k}^{*}(i)\right|^{2}+\sigma_{w}^{2}},
$$

where $\lambda_{k}(i)$ is the i-th element of the diagonal matrix $\boldsymbol{\Delta}_{\boldsymbol{k}}$. Finally, it is worth mentioning that the recursive least square (RLS) algorithm is used to update the MMSE filter matrix.

\section{B. Time Domain Equalizer (TDE)}

In practice, the channel may vary over the symbol block and the channel estimation is not completely accurate, leading to residual ISI after frequency equalization. After inverse Fourier transform, in the time domain, the FDE output symbol can be modeled as:

$$
\tilde{z}_{k}(n)=\tilde{h}_{0} s_{n}+\sum_{l \neq 0} \tilde{h}_{l} s_{n-l}+\tilde{w}(n)
$$

Thus, some additional time equalization is often necessary. We now describe the joint phase synchronizer and 
Feedforward-Feedback time domain equalizer depicted in Fig. 4. The proposed TDE consists of two equalizers : Feedforward $\left(f_{k}(n)\right.$ coefficients) and Feedback $\left(g_{k}(n)\right.$ coefficients). In order to obtain a better system performance for time-varying channels, the equalizers perform in parallel phase estimation [32]-[35] and equalization; the various equalized symbols $e_{k}$ at the output of the equalizers are then averaged. Note that for the first iteration, only the Feedforward equalizer is used. Moreover, the extrinsic information provided by the channel decoder at iteration (p-1) is fed back to the proposed technique at the next iteration and is used to both compensate the channel uncertainties and perform the Feedback equalization in the time domain to get rid of the residual ISI. After $p_{\max }$ iterations, the receiver decodes the transmitted symbol based on the LLR values given by the channel decoder.

For each iteration $p$, the time-domain equalizer is fed by the observations obtained at the output of the DFE $\tilde{Z}_{k}$ and the estimated data $\bar{S}(n)$ obtained from the previous iteration $(p-1)$. For the sake of simplicity, the index $(p)$ is dropped; the output of the TDE can be written as:

$$
z_{k}(n)=f_{k}(n)^{T} \tilde{Z}_{k}(n) e^{-i \hat{\phi}_{k, n}}-g_{k}(n)^{T} \widetilde{S}(n),
$$

where $\widetilde{S}(n)=\left[\tilde{s}\left(n+L_{2}\right), \cdots \tilde{s}(n) \cdots, \tilde{s}\left(n-L_{2}\right)\right]^{T}$ and $\tilde{s}(n)$ is either a known (in the preamble) or estimated symbol; $\tilde{Z}_{k}(n)=\left[\tilde{z}_{k}\left(n+L_{1}\right), \cdots \tilde{z}_{k}(n), \cdots, \tilde{z}_{k}\left(n-L_{1}\right)\right]^{T}$ denotes the received observations after FDE, $f_{k}(n)$ (resp. $\left.g_{k}(n)\right)$ is a transversal filter of length $2 L_{1}+1$ (resp. $\left.2 L_{2}+1\right)$, and $\hat{\Phi}_{k, n}$ is the estimated phase error. The coefficients of the filters are updated in order to minimize the estimated Mean-Square Error:

$$
J\left(f_{k}, g_{k}, \hat{\Phi}_{k}\right)=E\left\{\left|z_{k}(n)-\tilde{s}(n)\right|^{2}\right\} .
$$

Using the descent gradient algorithm, the TDE coefficients are updated as:

$$
\begin{aligned}
f_{k}(n+1) & =f_{k}(n)-\mu \nabla_{f_{k}}\left(\left|z_{k}(n)-\tilde{s}(n)\right|^{2}\right) \\
g_{k}(n+1) & =g_{k}(n)-\mu \nabla_{g_{k}}\left(\left|z_{k}(n)-\tilde{s}(n)\right|^{2}\right) \\
\hat{\Phi}(n+1) & =\hat{\Phi}(n)-\gamma \nabla_{\hat{\Phi}_{k}}\left(\left|z_{k}(n)-\tilde{s}(n)\right|^{2}\right),
\end{aligned}
$$

where $\mu$ and $\gamma$ are appropriate step sizes.

Finally, it is worth noting that in contrast to previous works, we propose to perform on each received channel symbols both FDE and TDE, and we also propose to perform jointly adaptive phase estimation and time domain equalization as this leads to better performance over difficult multipath channels as shown in the next section.

\section{Simulation Results}

In this section, we provide our simulation results in order to characterize our hybrid turbo equlization technique. Our proposed algorithm is tested over a real UWA channel measured off the commercial harbour of Brest, France (BCH1), over a distance of $800 \mathrm{~m} \mathrm{[31].} \mathrm{The} \mathrm{number} \mathrm{of} \mathrm{receiver} \mathrm{hydrophones}$

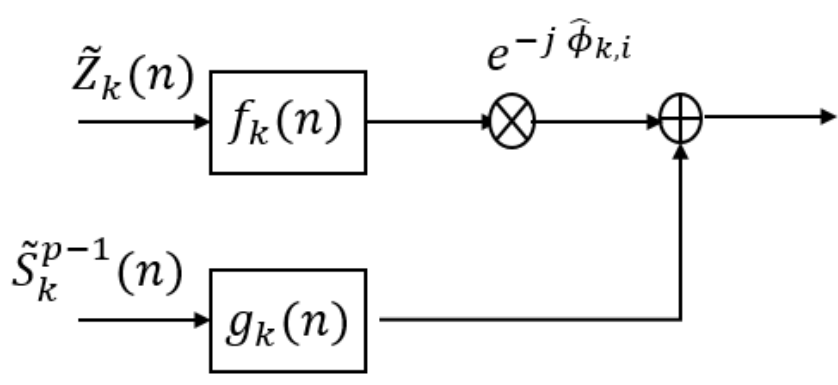

Fig. 3. Feedforward-Feedback time domain equalizer.

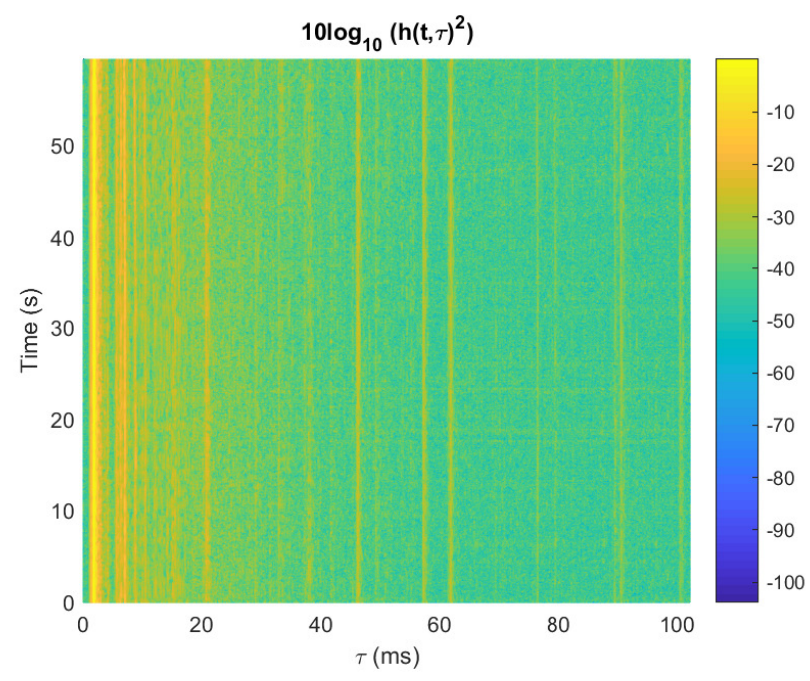

Fig. 4. The multi-path BCH1 channel.

is equal to 4. Fig. 4 describes the multi-path delay spread over this channel for the first hydrophone.

Moreover, in our simulations, user frames of 1000 bits are coded with a turbo code of rate $1 / 3$. The QPSK constellation is used. The block (resp. PN) length is 512 (resp. 128). The Feedforward (resp. Feedback) time filter length is fixed to 8 (resp. to 4) and $p_{\max }$ is fixed to 5. Figure 5 compares the BER performance between the multi-channel frequency domain equalizer (MFDE), the multi-channel time domain equalizer (MTDE), the hybrid time-frequency domain equalizer (HTFDE) [21] and the proposed method. With the MTDE method, the Feedforward (resp. Feedback) time filter length is fixed to 31 (resp. to 21). It can be observed that our proposal achieves the best BER performance among the considered methods. In particular, it achieves a gain of about $0.7 \mathrm{~dB}$ compared to HTFDE [21]. It also achieves a gain of about $0.5 \mathrm{~dB}$ compared to MTDE while reducing considerably the computational complexity.

Finally, in addition to the Watermark real recorded channel, we also perform simulation on a synthetic channel. As in [11], [36], channel coefficients are modeled as:

$$
h_{l}(k)=\sqrt{\frac{P_{l}}{I}} \sum_{i=1}^{I} e^{j\left(k \epsilon \pi \cos \psi_{l, i}+\zeta_{l, i}\right)},
$$




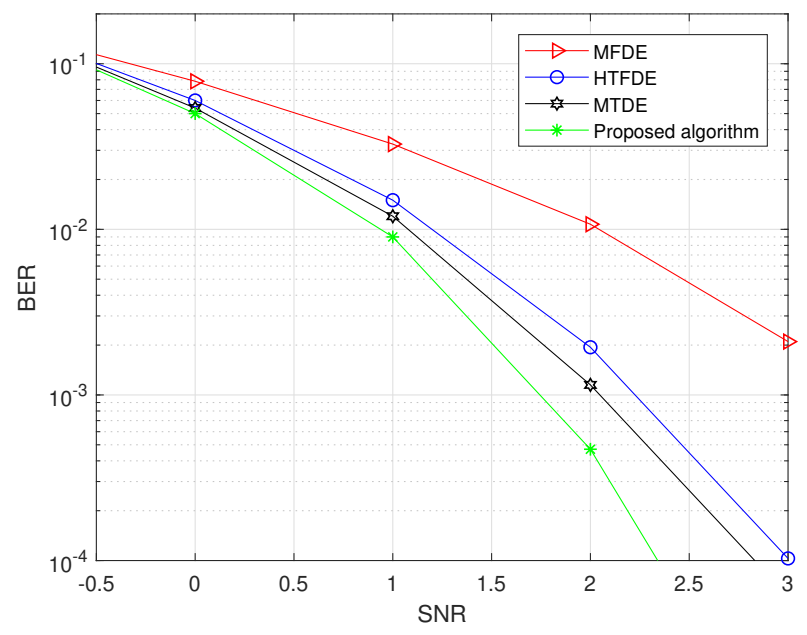

Fig. 5. BER comparison between the considered equalization algorithms over the BCH1 channel [31].

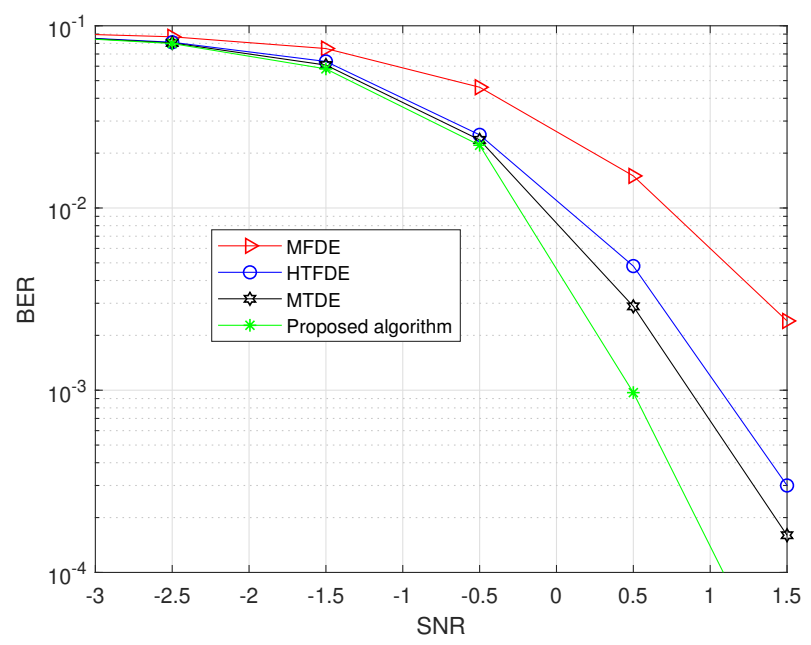

Fig. 6. BER comparison between the considered equalization algorithms over the channel (17), for the QPSK constellation and with 4 receive antennas.

where the Doppler shift is characterised by $\epsilon, P_{l}$ denotes the mean power of the $l$-th channel path, $\psi_{l, i}$ and $\zeta_{l, i}$ are uniform random variables over $[0,2 \pi[; I$ is fixed to 10 and (17) has three paths having all the same mean power in our simulation and $\epsilon$ is fixed to 0.002 .

Figure 6 compares the BER performance between the considered algorithms over the channel given by (17). We can observe again that our proposal achieves the best BER performance between the considered algorithms. In particular, it outperforms the conventional HTFDE by roughly $0.6 \mathrm{~dB}$.

\section{CONCLUSION}

This paper proposes a new hybrid turbo equalizer for FTN UWA communications systems. With the proposed algorithm, the receiver first equalizes the received symbol in the frequency domain. Thereafter, joint phase synchronisation and equalization are performed in the time domain on each channel. Simulation results of our proposal shows a significant BER improvement compared to conventional solutions. In particular, our proposal achieves a considerable gain in BER while reducing the computational complexity compared to the conventional MTDE, especially for a long-tap FTN scenario.

\section{REFERENCES}

[1] D. B. Kilfoyle and A. B. Baggeroer," The state of the art in underwater acoustic telemetry," IEEE Journal of Oceanic Engineering, 25(1) : 4-27, Jan. 2000

[2] Z. Ye, T. Arbi, F. X. Socheleau and B. Geller, "Fast Soft Demapping for Underwater Acoustic Communications With Signal Space Diversity," OCEANS 2018 MTS/IEEE Charleston, 2018, pp. 1-6, doi: 10.1109/OCEANS.2018.8604747.

[3] T. Arbi, Z. Ye and B. Geller, "Low-Complexity Blind PAPR Reduction for OFDM Systems With Rotated Constellations," IEEE Transactions on Broadcasting, vol. 67, no. 2, pp. 491-499, June 2021, doi: 10.1109/TBC.2021.3056232.

[4] T. Arbi, B. Geller, J. Yang, C. Abdel Nour and O. Rioul, "Uniformly Projected RCQD QAM: A Low-Complexity Signal Space Diversity Solution Over Fading Channels With or Without Erasures," IEEE Transactions on Broadcasting, vol. 64, no. 4, pp. 803-815, Dec. 2018, doi: 10.1109/TBC.2018.2811618.

[5] T. Arbi and B. Geller, "Joint BER Optimization and Blind PAPR Reduction of OFDM Systems With Signal Space Diversity," IEEE Communications Letters, vol. 23, no. 10, pp. 1866-1870, Oct. 2019 , doi: 10.1109/LCOMM.2019.2931898.

[6] T. Arbi, I. Nasr and B. Geller, "Near Capacity RCQD Constellations for PAPR Reduction of OFDM Systems," Proc. of IEEE International Conference on Acoustics, Speech and Signal Processing (ICASSP), pp. 5110-5114, May 2020, doi: 10.1109/ICASSP40776.2020.9053923

[7] J. Yang, K. Wan, B. Geller, C. Abdel Nour, O. Rioul and C. Douillard, "A low-complexity 2D signal space diversity solution for future broadcasting systems," 2015 IEEE International Conference on Communications (ICC), pp. 2762-2767, 2015, doi: 10.1109/ICC.2015.7248744.

[8] J. E. Mazo, "Faster-than-Nyquist signaling," Bell Syst. Technical J., vol. 54, no. 8, pp. 1451-1462, Oct. 1975.

[9] J. B. Anderson, F. Rusek, and V. Owall, "Faster than Nyquist signaling," Proc. IEEE, vol. 101, no. 8, pp. 1817-1830, Aug. 2013.

[10] J. G. Proakis, Digital Communications, 5th ed. McGraw-Hill, 2008.

[11] Z. Ye, T. Arbi, and B. Geller, "Low complexity adaptive turbo equalization for multipath underwater communication," Proceedings of IEEE Oceans 19, Marseille, June 2019.

[12] Junyi Xi, Shefeng Yan, Lijun Xu, Chaohuan Hou, "Sparsity-Aware Adaptive Turbo Equalization for Underwater Acoustic Communications in the Mariana Trench", IEEE Journal of Oceanic Engineering, vol. 46, no. 1, pp. 338-351, May 2021.

[13] A. Prlja and J. B. Anderson, "Reduced-complexity receivers for strongly narrowband intersymbol interference introduced by faster-than-Nyquist signaling," IEEE Trans. Commun., 60 (9), pp. 2591-2601, Sep. 2012.

[14] L. Zhou, B. Zheng, A. Wei, B. Geller and J. Cui, "A Robust ResolutionEnhancement Scheme for Video Transmission Over Mobile Ad-Hoc Networks," in IEEE Transactions on Broadcasting, vol. 54, no. 2, pp. 312-321, June 2008, doi: 10.1109/TBC.2008.917746.

[15] B. Geller, V. Capellano, J.-M. Brossier, A. Essebbar and G. Jourdain, "Equalizer for video rate transmission in multipath underwater communications," IEEE Journal of Oceanic Engineering, vol. 21, no. 2, pp. 150-155, April 1996, doi: 10.1109/48.486790.

[16] B. Geller, V. Capellano and G. Jourdain, "Equalizer for real time high rate transmission in underwater communications," 1995 International Conference on Acoustics, Speech, and Signal Processing, 1995, pp. 3179-3182 vol.5, doi: 10.1109/ICASSP.1995.479560.

[17] B. Geller, V. Capellano, J.M. Broissier, "Equalizer for High Rate Transmission," Proc. of OCEANS IEEE Oceanic engineering, pp. 302306, Brest, Sept. 1994

[18] Y. R. Zheng, J. Wu and C. Xiao, ”Turbo equalization for single-carrier underwater acoustic communications", IEEE Commun. Mag., vol. 53, no. 11, pp. 79-87, Nov. 2015. 
[19] S. Sugiura, "Frequency-Domain Equalization of Faster-than-Nyquist Signaling," IEEE Wireless Communications Letters, vol. 2, no. 5, pp. 555-558, October 2013.

[20] Z. Chen, J. Wang and Y. R. Zheng, ’Frequency-Domain Turbo Equalization With Iterative Channel Estimation for MIMO Underwater Acoustic Communications," IEEE Journal of Oceanic Engineering, vol. 42, no. 3, pp. 711-721, July 2017.

[21] Junyi Xi, Shefeng Yan, Lijun Xu, Zhen Zhang, Di Zeng "Frequency-Time Domain Turbo Equalization for Underwater Acoustic Communications", IEEE Journal of Oceanic Engineering 45: 665-679, April 2020.

[22] C. B. He, J. G. Huang, Q. F. Zhang, "Hybrid Time-Frequency Domain Equalization for Single-Carrier Underwater Acoustic Communications" WUWNet'12, Nov.5-6, 2012, Los Angeles, California, USA.

[23] C. He, S. Huo, H. Wang, Q. Zhang and J. Huang, "Single carrier with multi-channel time-frequency domain equalization for underwater acoustic communications," 2015 IEEE International Conference on Acoustics, Speech and Signal Processing (ICASSP), South Brisbane, QLD, Australia, pp. 3009-3013, 2015.

[24] Chengbing He, Lianyou Jing, Rui Xi, Han Wang, Fei Hua, Qianqian Dang, Qunfei Zhang, "Time-Frequency Domain Turbo Equalization for Single-Carrier Underwater Acoustic Communications", Access IEEE, vol. 7, pp. 73324-73335, May 2019.

[25] C. Berrou, A. Glavieux and P. Thitimajshima, "Near Shannon limit errorcorrecting coding and decoding: Turbo-codes," Proceedings of ICC '93 - IEEE International Conference on Communications, 1993, pp. 10641070 vol.2, doi: 10.1109/ICC.1993.397441.

[26] B. Geller, I. Diatta, J. P. Barbot, C. Vanstraceele and F. Rambeau, 'Block Turbo Codes: From Architecture to Application," IEEE International Symposium on Information Theory, ISIT 2006, pp. 1813-1816, July 2006, doi: 10.1109/ISIT.2006.261747.

[27] I. Nasr, B. Geller, L. Najjar Atallah, S. Cherif, "Performance Study of a
Near Maximum Likelihood Code-Aided Timing Recovery Technique," IEEE Transactions on Signal Processing, 64(3), pp. 799-811, Feb. 2016.

[28] I. Nasr, L. N. Atallah, S. Cherif and B. Geller, "Near MAP Dynamical Delay Estimator and Bayesian CRB for Coded QAM Signals," IEEE Transactions on Wireless Communications, vol. 17, no. 1, pp. 636-651, Jan. 2018, doi: 10.1109/TWC.2017.2769043.

[29] J. Yang, B. Geller and T. Arbi, "Proposal of a multi-standard transceiver for the WBAN Internet of Things," 2016 International Symposium on Signal, Image, Video and Communications (ISIVC), pp. 369-373, Nov. 2016, doi: 10.1109/ISIVC.2016.7894017.

[30] T. Arbi, J. Yang, B. Geller, "Récepteur multi-normes pour les réseaux de capteurs de l'iot médical," Internet des objets, 1 (Numéro 1), 2017.

[31] P. A. van Walree, F. X. Socheleau, R. Otnes, and T. Jenserud, "The "watermark" benchmark for underwater acoustic modulation schemes," IEEE Journal of Oceanic Engineering, 42(4), pp. 1007-1018, Oct. 2017.

[32] J. Yang, B. Geller and S. Bay, 'Bayesian and Hybrid Cramér-Rao Bounds for the Carrier Recovery Under Dynamic Phase Uncertain Channels," IEEE Transactions on Signal Processing, vol. 59, no. 2, pp. 667-680, Feb. 2011, doi: 10.1109/TSP.2010.2081981.

[33] J. Yang, B. Geller and A. Wei, "Approximate Expressions for CramerRao Bounds of Code Aided QAM Dynamical Phase Estimation," 2009 IEEE International Conference on Communications, 2009, pp. 1-5, doi: 10.1109/ICC.2009.5198756.

[34] J. Yang and B. Geller, "Near Optimum Low Complexity Smoothing Loops for Dynamical Phase Estimation-Application to BPSK Modulated Signals," IEEE Transactions on Signal Processing, vol. 57, no. 9, pp. 3704-3711, Sept. 2009, doi: 10.1109/TSP.2009.2021452.

[35] J.M. Brossier, P.O. Amblard, B. Geller, "Self-adaptive PLL for general QAM constellations," 2002 11th European Signal Processing Conference, Proc. EUSIPCO, pp. 631-635, Sept. 2002.

[36] C. Laot, A. Glavieux, and J. Labat, " Turbo equalization: adaptive equalization and channel decoding jointly optimized," IEEE Journal on Selected Areas in Communications, 19, pp. 1744-1752, Sep 2001. 